\title{
Lengthscale dependence of dynamic four-point susceptibilities in glass formers
}

\author{
David Chandler, ${ }^{1}$ Juan P. Garrahan, ${ }^{2}$ Robert L. Jack, ${ }^{1}$ Lutz Maibaum, ${ }^{1}$ and Albert C. Pan ${ }^{1}$ \\ ${ }^{1}$ Department of Chemistry, University of California, Berkeley, CA 94720-1460 \\ ${ }^{2}$ School of Physics and Astronomy, University of Nottingham, Nottingham, NG7 2RD, UK
}

\begin{abstract}
Dynamical four-point susceptibilities measure the extent of spatial correlations in the dynamics of glass forming systems. We show how these susceptibilities depend on the length scales that necessarily form part of their definition. The behaviour of these susceptibilities is estimated by means of an analysis in terms of renewal processes within the context of dynamic facilitation. The analytic results are confirmed by numerical simulations of an atomistic model glass-former, and of two kinetically constrained models. Hence we argue that the scenario predicted by the dynamic facilitation approach is generic.
\end{abstract}

\section{INTRODUCTION}

The length scales governing dynamical heterogeneity in glass-forming liquids [1, 2, 3, 4, 5] are often described in terms of the susceptibility associated with fluctuations in the self intermediate scattering function [4, 6, 7, 8, 9]:

$$
\chi_{4}(k, t) \equiv \frac{1}{N} \sum_{j l}\left\langle\delta \hat{F}_{j}(\boldsymbol{k}, t) \delta \hat{F}_{l}(-\boldsymbol{k}, t)\right\rangle .
$$

Here the indices $j$ and $l$ run over the $N$ particles in the system, the position of the $j$ th particle at time $t$ is $\hat{\boldsymbol{r}}_{j}(t)$;

$$
\delta \hat{F}_{j}(\boldsymbol{k}, t) \equiv e^{i \boldsymbol{k} \cdot\left[\hat{\boldsymbol{r}}_{j}(t)-\hat{\boldsymbol{r}}_{j}(0)\right]}-\left\langle e^{i \boldsymbol{k} \cdot\left[\hat{\boldsymbol{r}}_{j}(t)-\hat{\boldsymbol{r}}_{j}(0)\right]}\right\rangle,
$$

and $k=|\boldsymbol{k}|$. Under supercooled conditions, this fourpoint correlation function typically grows in time towards a peak, before decreasing at large times. This non-monotonic behaviour is a consequence of the transient nature of dynamic heterogeneity.

It was suggested by Toninelli et al. 8] that the time dependence of $\chi_{4}(k, t)$ can be used to distinguish between different theoretical scenarios for the glass transition. The dependence of $\chi_{4}(k, t)$ on the wave vector $k$ was considered for a glass-forming system in [10], and for a sheared granular material in [11]. In both cases, significant dependence on wave vector was found. In this article, we investigate this wave vector dependence, in particular the way that $\chi_{4}(k, t)$ grows towards its peak. We present data for an atomistic system, and for two kinetically constrained models [12]. We find non-trivial wave vector dependence in all three cases. We explain this generic behaviour analytically using a treatment that we used earlier to describe dynamic decoupling in glass formers $13,14,15]$.

Our analysis shows that the non-trivial behaviour of four-point correlators comes from two sources. The first contribution arises because particles that have not moved are clustered in space; the second comes from correlations between particle displacements. The relative sizes of these contributions depend on the wave vector $k$. The first dominates when $k$ is large, and the second dominates when $k$ is small. The crossover between these two regimes corresponds to the crossover between non-Fickian and Fickian regimes observed in two-point functions [15]. (a)

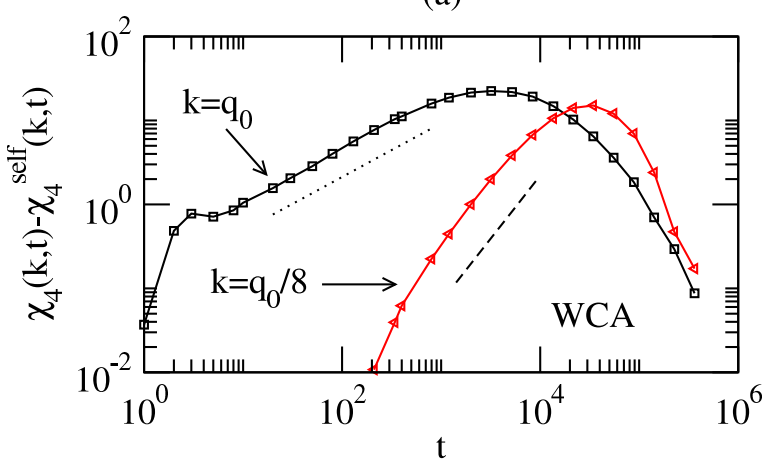

(b)

(c)

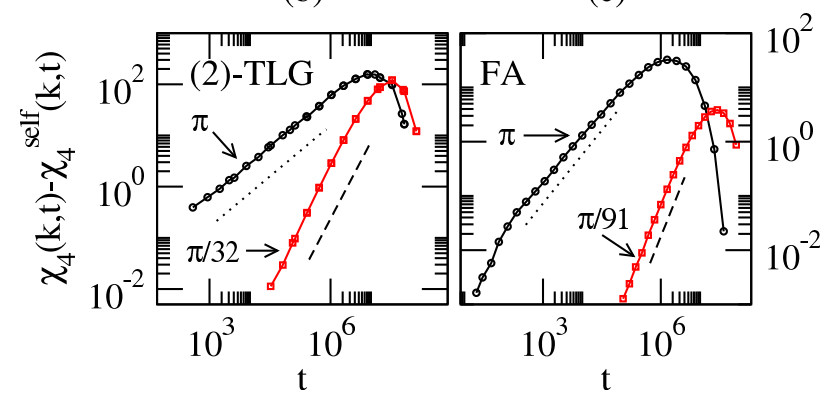

FIG. 1: (Color online) The distinct part of $\chi_{4}(k, t)$ as a function of $t$ for two wave vectors in three model systems: the supercooled three dimensional WCA mixture (a); a kinetically constrained triangular lattice gas (b); and probes in the one dimensional Fredrickson-Andersen model (c). The symbol $\chi_{4}^{\text {self }}(k, t)$ denotes $\left\langle\left|\delta \hat{F}_{i}(k, t)\right|^{2}\right\rangle$. Full details are presented in the relevant sections below. Dashed and dotted lines show that in all cases the exponents of power law fits increase with decreasing wave vector. The largest wave vectors considered are the principal wave vectors for each system (for the WCA case this is the peak location in the equilibrium structure factor, $\left.q_{0}\right)$.

Figure 1 illustrates the behaviour that we consider for three different model systems. These systems, described in detail in sections III and IV. represent three levels of coarse-graining in the glassy system. The most detailed is a fluid mixture of classical particles in continuous three dimensional space, interacting with WeeksChandler-Andersen (WCA) potentials [16, 17]. The sec- 
ond is the so-called (2)-TLG, due to Jäckle and Krönig [18]. It is a kinetically constrained lattice gas in which the dynamics are highly co-operative and relaxation times increase very quickly with increasing density. The final system is the one dimensional one spin facilitated Fredrickson-Andersen (FA) model [19] to which probe particles have been added, following Ref. [13]. The probe particles do not interact with one another, but they propagate through an environment that is dynamically heterogeneous. In this model, all of the atomistic interactions have been removed, leaving only an idealised dynamically heterogeneous system.

The similarity of $\chi_{4}(k, t)$ between these three different model systems is striking. The four-point susceptibility has the usual single peak, and the increasing part of each curve can be fitted by a power law. In all three cases, the exponent with which $\chi_{4}(k, t)$ increases depends strongly on $k$. Further, all cases exhibit a shift of the peak of $\chi_{4}(k, t)$ to later times as $k$ decreases.

\section{THEORY OF FOUR-POINT FUNCTIONS IN HETEROGENEOUS SYSTEMS}

\section{A. The dynamical facilitation approach}

Our starting point is to follow [15] and write

$$
\begin{aligned}
\hat{F}_{j}(\boldsymbol{k}, t) & \equiv \exp \left[i \boldsymbol{k} \cdot \Delta \hat{\boldsymbol{r}}_{j}(t)\right] \\
& =\hat{p}_{j}(t)+\left[1-\hat{p}_{j}(t)\right] \exp \left[i \boldsymbol{k} \cdot \Delta \hat{\boldsymbol{r}}_{j}(t)\right],
\end{aligned}
$$

where $\Delta \hat{\boldsymbol{r}}_{j}(t) \equiv \hat{\boldsymbol{r}}_{j}(t)-\hat{\boldsymbol{r}}_{j}(0)$ and $\hat{p}_{j}(t)$ is the local persistence operator. That is, $\hat{p}_{i}(t)$ takes the value of unity if particle $j$ has not moved a distance greater than some microscopic cutoff $a_{0}$, and it is zero otherwise. We use hats throughout this article to denote fluctuating quantities (operators).

The usefulness of (3) lies in the fact that the two terms separate mobile and immobile particles, explicitly accounting for the dynamical heterogeneity in the system. The expectation of each term can be simply evaluated in an appropriate Gaussian, or homogeneous, approximation, leading to:

$$
\begin{aligned}
F_{\mathrm{s}}(k, t) & \equiv\left\langle\hat{F}_{j}(\boldsymbol{k}, t)\right\rangle \\
& \approx P(t)+[1-P(t)] \exp \left(-k^{2} D t\right),
\end{aligned}
$$

where $P(t)$ is the average persistence function $P(t) \equiv$ $\left\langle\hat{p}_{j}(t)\right\rangle$ and $D$ is the self-diffusion constant. The approximate equality is valid [15] in the deeply supercooled regime of large decoupling between $\alpha$-relaxation time and diffusion rate [20]. According to this approximation, particle motion is a random walk with randomly distributed waiting times [21].

Moving from two point to four point functions, we consider the correlator $G_{j l}(k, t)=\left\langle\delta \hat{F}_{j}(\boldsymbol{k}, t) \delta \hat{F}_{l}(-\boldsymbol{k}, t)\right\rangle$ where $\delta \hat{F}_{j}=\hat{F}_{j}-F_{\mathrm{s}}$ was defined above. The diagonal (self) part is:

$$
G_{j j}(k, t)=1-F_{\mathrm{s}}(k, t)^{2} .
$$

It grows monotonically with time and is always smaller than unity. Non-trivial spatial correlations of the dynamics appear in the off-diagonal terms $j \neq l$. Using Eqs. (3) and (4), these correlations are

$$
\begin{aligned}
G_{j l}(k, t) \approx & \left(1-e^{-k^{2} D t}\right)^{2}\left\langle\delta \hat{p}_{j}(t) \delta \hat{p}_{l}(t)\right\rangle \\
& +[1-P(t)]^{2}\left\langle\delta e^{i \boldsymbol{k} \cdot \Delta \hat{\boldsymbol{r}}_{j}(t)} \delta e^{-i \boldsymbol{k} \cdot \Delta \hat{\boldsymbol{r}}_{l}(t)}\right\rangle
\end{aligned}
$$

where $\delta \hat{p}_{j}=\hat{p}_{j}-P$ and $\delta e^{i \boldsymbol{k} \cdot \Delta \hat{\boldsymbol{r}}_{j}(t)}=e^{i \boldsymbol{k} \cdot \Delta \hat{\boldsymbol{r}}_{j}(t)}-e^{-k^{2} D t}$. We have dropped terms that are cubic and quartic in the fluctuations since we expect their contributions to be small.

To arrive at the four-point susceptibility, we must sum over $j$ and $l$. The first term of (6) is the susceptibility of the persistence, and we have

$$
\frac{1}{N} \sum_{j l}\left\langle\delta \hat{p}_{j}(t) \delta \hat{p}_{l}(t)\right\rangle \approx N_{\mathrm{p}}(t) P(t)[1-P(t)]
$$

Here we have assumed that excitations propagate through the system leading to movement of particles [13], and that the average number of particles visited by a single excitation in time $t$ is $N_{\mathrm{p}}(t)$. This number is related to the dynamic correlation length of relaxed clusters of particles through an exponent representing their (possibly fractal) dimensionality. $N_{\mathrm{p}}(t)$ is also is related to the average persistence: a molecule will typically persist after time $t$ if there were no excitations initially in a surrounding region of mass $N_{\mathrm{p}}(t)$; the probability for this to happen is $P(t) \approx e^{-c N_{\mathrm{p}}(t)}$, where $c \ll 1$ is the average concentration of excitations. The calculation of the average of the product of the persistence is analogous: for $p_{j} p_{l}$ to be nonzero after time $t$ a mass $N_{\mathrm{p}}(t)$ has to be free of excitations initially around $j$ and $l$. The fact that these two volumes may overlap gives rise to spatial correlations between $p_{j}$ and $p_{l}$. Following similar arguments to those used in Ref. [8] one arrives to equation (77).

While the contribution to $\chi_{4}(k, t)$ from equation (7) measures whether nearby particles relax in a correlated way, the second term of (6) measures correlations in the displacements of particles that have relaxed. We define the correlation function for particle displacements in the following way:

$$
g(r, t) \equiv t^{-1}\left\langle\Delta \hat{\boldsymbol{r}}_{j}(t) \cdot \Delta \hat{\boldsymbol{r}}_{l}(t)\right\rangle_{r_{j l}=r}
$$

where the average is conditioned on the initial separation of the particles $r_{j l} \equiv\left|\boldsymbol{r}_{j}(0)-\boldsymbol{r}_{l}(0)\right|$. This function $g(r, t)$ measures the correlations between displacements of nearby particles. For simple Brownian motion, the separation of a pair of particles diffuses four times as fast as their centre of mass. If $g(r, t)$ is positive, then the separation diffuses more slowly than this reference value; if it is negative then the separation diffuses faster. If two particles move through the system facilitated by the same excitation then their separation remains relatively small, while their centre of mass moves a long way. We 

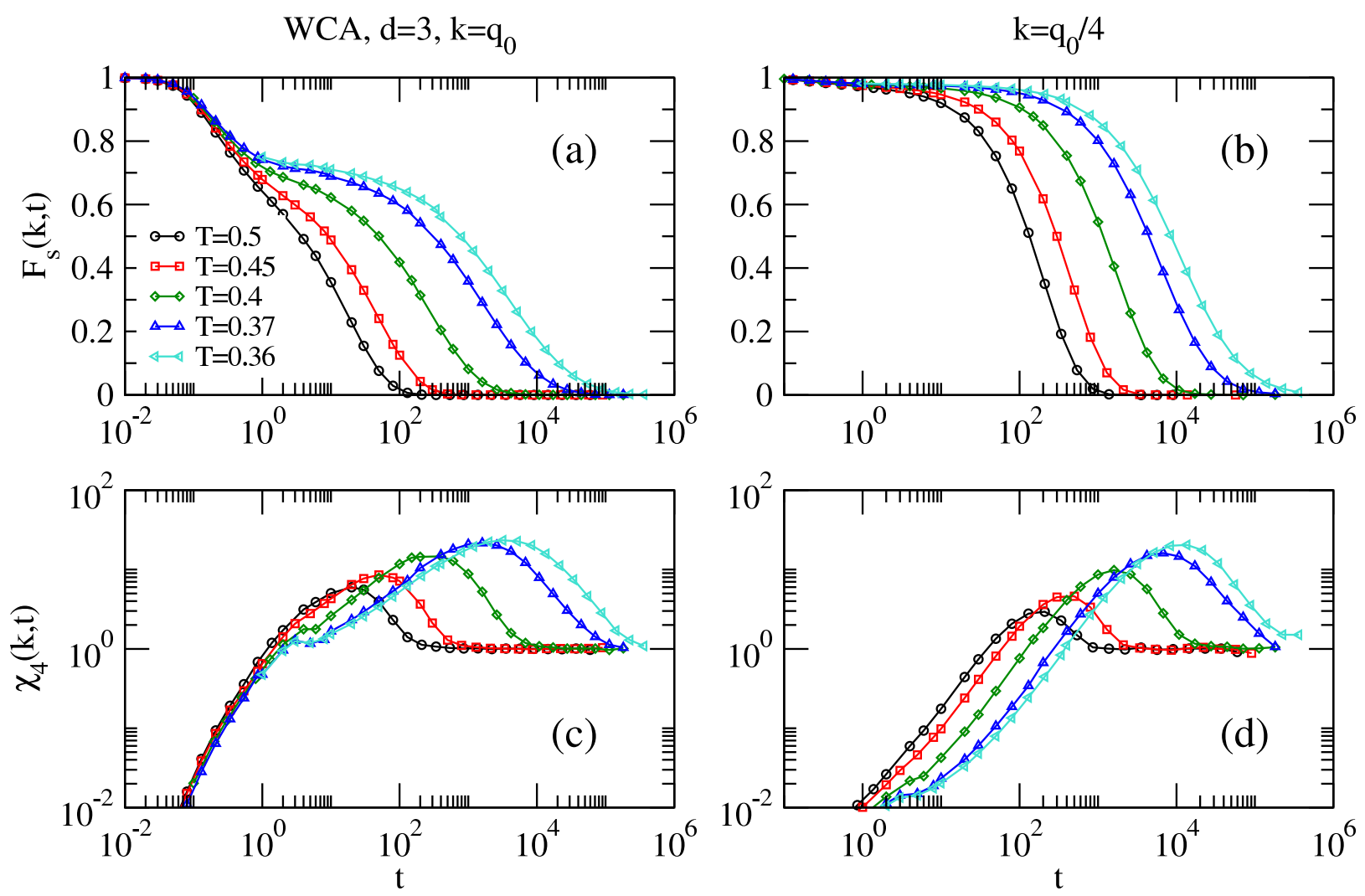

FIG. 2: (Color online) (a,b) Self-intermediate scattering functions $F_{s}(k, t)$ in the WCA mixture for wave vectors $k=q_{0}$ and $k=q_{0} / 4$ ( $q_{0}$ is the wave vector of the first peak in the structure factor). (c,d) Corresponding four-point susceptibilities $\chi_{4}(k, t)$.

show the resulting positive $g(r, t)$ for a simple kinetically constrained model in Fig 6 (below).

Within the Gaussian approximation, we arrive at

$\left\langle\delta e^{i \boldsymbol{k} \cdot \Delta \boldsymbol{r}_{j}(t)} \delta e^{-i \boldsymbol{k} \cdot \Delta \hat{\boldsymbol{r}}_{l}(t)}\right\rangle_{r_{j l}=r} \approx e^{-2 k^{2} D t}\left(e^{2 t k^{2} g(r, t)}-1\right)$

The Gaussian approximation is justified because particles have made many diffusive steps. The sum over particles at a given time $t$ is dominated by pairs of particles whose initial separation coincides with the maximum of $g(r, t)$. This leads to a contribution to $\chi_{4}(k, t)$ of

$$
\begin{aligned}
\frac{1}{N} \sum_{j l} & \left\langle\delta e^{i \boldsymbol{k} \cdot \Delta \hat{\boldsymbol{r}}_{j}(t)} \delta e^{-i \boldsymbol{k} \cdot \Delta \hat{\boldsymbol{r}}_{l}(t)}\right\rangle \\
& \approx N_{g}(t) e^{-2 k^{2} D t}\left(e^{2 t k^{2} \bar{g}(t)}-1\right),
\end{aligned}
$$

where $\bar{g}(t)=\max _{x}[g(x, t)]$ obeys $\bar{g}(t)<D$ and decreases at large times. We have assumed that the sum over $j$ is dominated by particles for which $g(r, t) \simeq \bar{g}(t)$, and we denote the number of these particles by $N_{g}(t)$. The summand is exponential in $t g(r, t)$, so this is a good approximation when that function is large. In the excitation picture we only expect strong displacement correlations when two particles are facilitated by the same excitation, so we expect $N_{g}(t)<N_{p}(t)$ in general.
Putting the results of Eqs. (5)-(77) and (9) together we arrive at the four-point susceptibility,

$$
\begin{aligned}
\chi_{4}(k, t) \approx & N_{\mathrm{p}}(t) P(t)[1-P(t)]\left(1-e^{-k^{2} D t}\right)^{2} \\
& +N_{g}(t)[1-P(t)]^{2} e^{-2 k^{2} D t}\left(e^{2 t k^{2} \bar{g}(t)}-1\right) \\
& +1-F_{\mathrm{s}}(k, t)^{2} .
\end{aligned}
$$

Thus, correlations between particles come from persistence correlations [first term in (10)] and from displacement correlations [second term in [10)]. Both contributions are non-monotonic in time. The persistence contribution peaks at a time $t_{\text {peak }}$ that scales as $t_{\text {peak }} \sim \tau_{\alpha}$, where $\tau_{\alpha}$ is the structural relaxation time. This term is relevant if $k$ is large. Conversely, the second term in (10) dominates at small $k$, when $D k^{2} \tau_{\alpha} \ll 1$. The peak of this term occurs at $t_{\text {peak }} \sim\left(D k^{2}\right)^{-1}$, and so increases with decreasing $k$, while its peak height decreases as $\bar{g}\left(t_{\text {peak }}\right)$.

Equation (10) is consistent with the data of Fig. 11 As well as the overall form of $\chi_{4}$, it contains two main predictions. Firstly, for a given wave vector, $\chi_{4}(k, t)$ peaks at a $k$-dependent time $t_{\text {peak }}$. The scaling of this time is the same as that of the time scale of two-point correlators [15]. It increases with decreasing $k$, consistent with simulations. A corollary is that $\chi_{4}(k, t)$ may display a non-trivial structure even for wavelengths at which the corresponding one-particle motion is Fickian. 

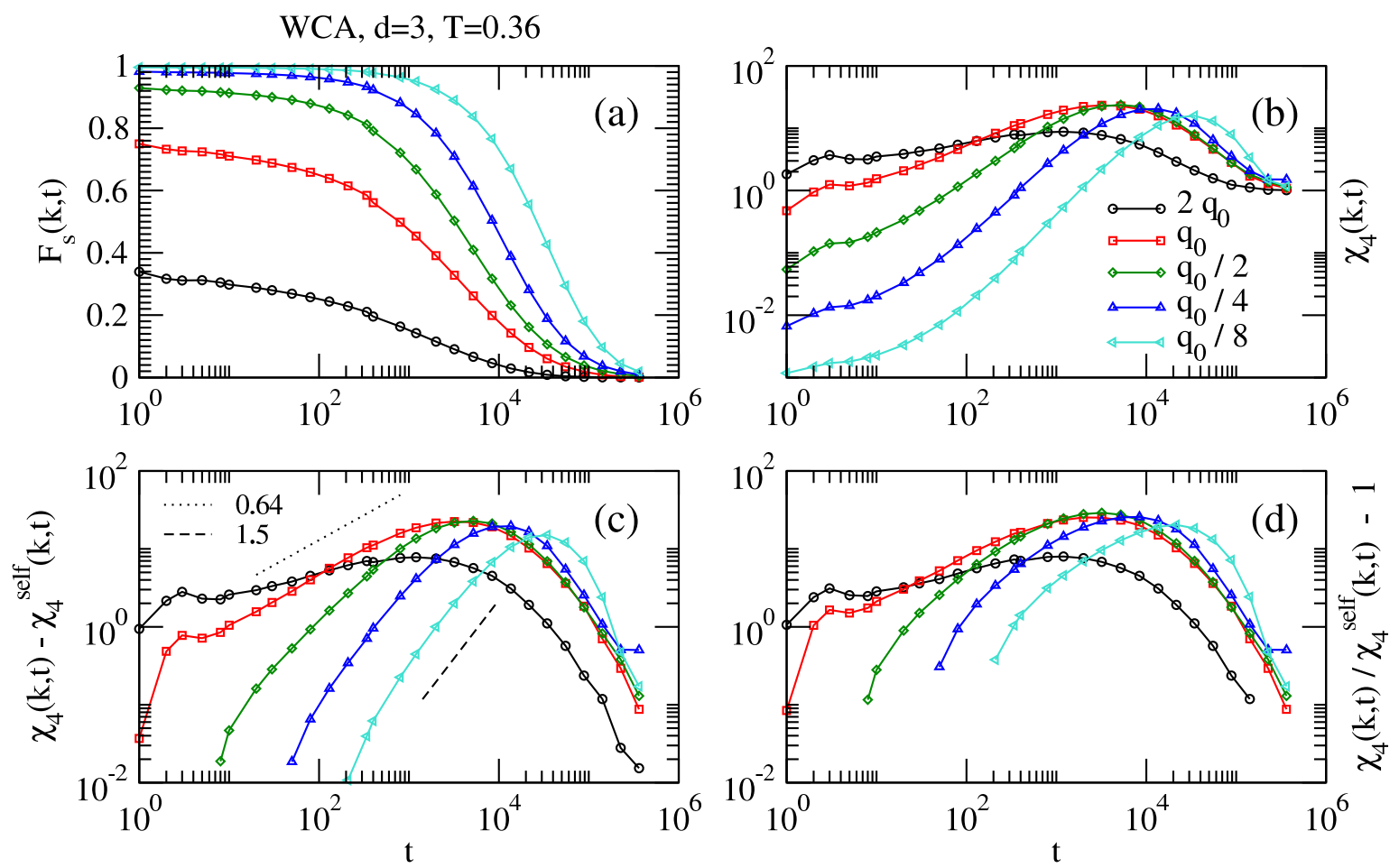

FIG. 3: (Color online) (a) Self-intermediate scattering functions $F_{s}(k, t)$ in the WCA mixture at $T=0.36$, for wave vectors $k=2 q_{0}, q_{0}, q_{0} / 2, q_{0} / 4, q_{0} / 8$. (b) Corresponding four-point susceptibilities $\chi_{4}(k, t)$. (c) Distinct part $\chi_{4}(k, t)-\chi_{4}^{\text {self }}(k, t)$. (d) Distinct part normalized by the self term, $\chi_{4}(k, t) / \chi_{4}^{\text {self }}(k, t)-1$.

Secondly, the increase of $\chi_{4}(k, t)$ depends on the wave vector $k$ : from above, we have for large $k$

$$
\chi_{4}(k, t) \sim N_{\mathrm{p}}(t)[1-P(t)], \quad D k^{2} t \gg 1,
$$

and for small $k$

$$
\chi_{4}(k, t) \sim N_{g}(t)[1-P(t)]^{2} \bar{g}(t) k^{2} t, \quad D k^{2} t \ll 1 .
$$

The two relations come from persistence (large $k$ ) and displacement correlations (small $k$ ). These two scaling predictions are different in general: we argue that this accounts for the variation with $k$ at early times that was demonstrated in figure 1. Within the simplest picture of facilitation, $N_{p}(t)$ is the fundamental object: it measures the number of particles whose relaxation is facilitated by a single mobility excitation. At this minimal level of theory, and for small times $\left(t<<\tau_{\alpha}\right)$, we expect the number of particles that have relaxed to be $N_{c} N_{p}(t)$, where $N_{c}$ is the number of mobility excitations; it follows that $[1-P(t)] \sim N_{p}(t)$. Of the $N_{p}$ particles facilitated by a single excitation, we expect a finite fraction to have strong displacement correlations: this finite fraction is $N_{g} / N_{p}$. If this assumption holds then $N_{g}(t)$ and $N_{p}(t)$ will have the same scaling with time and temperature.

Following Toninelli et al. [8], we define an exponent $\mu$ by $\chi_{4}(t) \sim t^{\mu}$. The observation of Ref. [8] is that $\mu \simeq b$, where $b$ is the von-Schweidler $\beta$-relaxation exponent of mode-coupling theory (MCT) 22]. Eq. (10) shows clearly that $\mu$ depends on wave vector, $\mu=\mu(k)$; however, $\mathrm{MCT}$ predicts a dynamical critical point at which $b$ is independent of $k$ [22] (see nevertheless the discussion in [23]). In supercooled liquids, no such phase transition is observed, and the system is always in the ergodic phase. Hence, all correlation lengths are finite, and the scaling relation $\mu \simeq b$ breaks down for wave vectors smaller than the inverse correlation length. This effect can be investigated by direct numerical solutions of the MCT equations [24, 25], but agreement with simulation is still poor for small wave vectors. This is consistent with the hypothesis that processes neglected by the mode coupling approximation are important for the structural relaxation of supercooled liquids, leading to avoidance of the dynamical transition; to the decoupling of diffusion and viscosity [25]; and to non-trivial wave vector dependence of two and four-point correlations on large length scales.

\section{B. Related four-point functions}

The function

$$
G_{4}(\boldsymbol{k}, \boldsymbol{q}, t)=\frac{1}{N} \sum_{j l}\left\langle\delta \hat{F}_{j}(\boldsymbol{k}, t) \delta \hat{F}_{l}(-\boldsymbol{k}, t) \mathrm{e}^{i \boldsymbol{q} \cdot\left[\hat{\boldsymbol{r}}_{j}(0)-\hat{\boldsymbol{r}}_{l}(0)\right]}\right\rangle
$$

generalizes $\chi_{4}(k, t)$. Clearly $\chi_{4}(k, t)=G_{4}(\boldsymbol{k}, 0, t)$. However, $G_{4}(\boldsymbol{k}, \boldsymbol{q}=0, t)$ is ensemble dependent (see e.g. [26]), whereas $\lim _{q \rightarrow 0} G_{4}(\boldsymbol{k}, \boldsymbol{q}, t)$ is not. For the WCA 

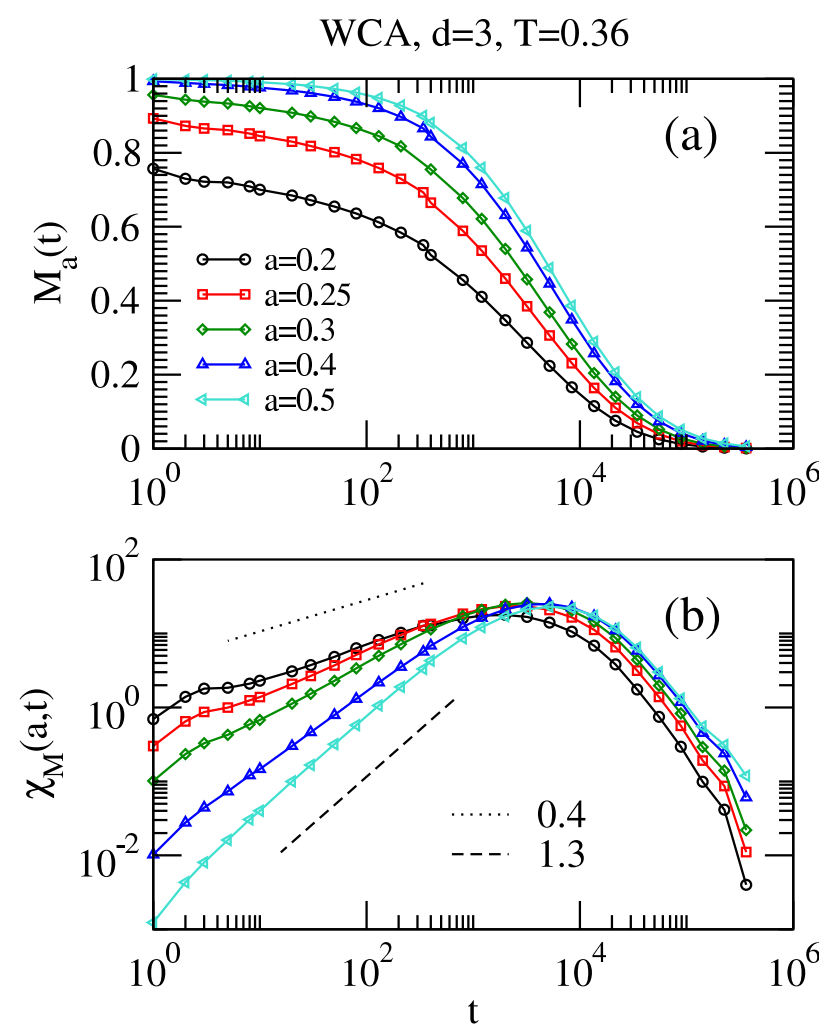

FIG. 4: (Color online) (a) Two point overlap correlators $M_{a}(t)$ in the WCA atomistic model at temperature $T=0.36$.

(b) Corresponding four point susceptibilities $\chi_{M}(a, t)$.

mixture and the (2)-TLG we have found that the ensemble dependence affects the absolute value of $\chi_{4}(k, t)$ but not their functional trends. The differences in $\chi_{4}(k, t)$ between ensembles can be calculated in terms of thermodynamic properties and derivatives of $F_{\mathrm{s}}(k, t)$ [27]. Berthier et al. [26] suggest that these differences provide reliable estimates of $\lim _{q \rightarrow 0} G_{4}(\boldsymbol{k}, \boldsymbol{q}, t)$ itself. For the WCA mixture and (2)-TLG, in the regimes accessible to our simulations, we find that these differences also have an important $k$ dependence, and that they only account for a fraction of the total value of $\lim _{q \rightarrow 0} G_{4}(\boldsymbol{k}, \boldsymbol{q}, t)$. This is discussed in detail in the Appendix.

An alternative (ensemble dependent) four point function is

$$
\chi_{M}(a, t) \equiv \frac{1}{N} \sum_{j l}\left\langle\delta \hat{M}_{j}(a, t) \delta \hat{M}_{l}(a, t)\right\rangle
$$

where $\delta \hat{M}_{i}=\hat{M}_{i}-\left\langle\hat{M}_{i}\right\rangle$ in which $\hat{M}_{i}(a, t)$ is a (binary) operator that equals unity if $\left|\hat{r}_{i}(t)-\hat{r}_{i}(0)\right|<a$ and zero otherwise.

The operator $\hat{M}(a, t)$ was used, for example, in [10]. Its ensemble average, $M_{a}(t) \equiv\left\langle\hat{M}_{j}(a, t)\right\rangle$, is the fraction of particles that have not moved beyond a distance $a$ in time $t$, and is related to the self-intermediate scattering function by $M_{a}(t)=(2 \pi)^{-d} \int_{|\boldsymbol{r}|<a} \mathrm{~d}^{d} \boldsymbol{r} \int \mathrm{d}^{d} \boldsymbol{k} e^{-i \boldsymbol{k} \cdot \boldsymbol{r}} F_{\mathrm{s}}(k, t)$.

Our analysis of $\chi_{4}(k, t)$ generalizes immediately to $\chi_{M}$ : we write

$$
\hat{M}_{j}(a, t)=\hat{p}_{j}(t)+\left[1-\hat{p}_{j}(t)\right] \Theta\left(a-\left|\Delta \hat{\boldsymbol{r}}_{j}(t)\right|\right),
$$

where $\Theta(x)$ is the Heaviside function. The difference between the operators $\hat{M}_{j}(a, t)$ and $\hat{F}_{j}(k, t)$ is that the phase of $\hat{F}_{j}(k, t)$ records the direction of motion of the $j$ th particle. For small $a$, correlations between the directions in which mobile particles have moved do not contribute to $\chi_{M}(a, t)$ and we expect it to be dominated by the persistence correlations:

$$
\chi_{M}(a, t) \approx f\left(D t / a^{2}\right) N_{\mathrm{p}}(t) P(t)[1-P(t)]
$$

where $f(x)=(4 \pi D t)^{-d}\left[\int_{|\boldsymbol{r}|<a} \mathrm{~d}^{d} \boldsymbol{r} e^{-|\boldsymbol{r}|^{2} /(D t)}\right]^{2}$. The structure is the same as the first term of (10) with $f\left(D t / a^{2}\right)$ playing the part of $\left(1-e^{-D k^{2} t}\right)^{2}$. We note also that $\chi_{M}(a, t)$ approaches the contribution of (7) as $a$ gets small, as expected. We compare this prediction with atomistic simulations in section III, see below.

\section{ATOMISTIC SIMULATIONS}

For a continuous atomistic representation of a supercooled liquid, we carried out extensive molecular dynamics simulations of a symmetric WCA mixture [16]. It is a mixture of two particle species $\mathrm{A}$ and $\mathrm{B}$ in three spatial dimensions. The potential energy is the sum of the pairwise interactions between two particles of species $\alpha$ and $\beta, V_{\alpha \beta}(r)=4 \varepsilon\left[\left(\sigma_{\alpha \beta} / r\right)^{12}-\left(\sigma_{\alpha \beta} / r\right)^{6}+1 / 4\right]$ if $r \leq 2^{1 / 6} \sigma_{\alpha \beta}$, and $V_{\alpha \beta}(r)=0$ otherwise. This is the reference potential of the WCA theory [17] consisting of the repulsive part of the Lennard-Jones interaction. Following [10, 28] we choose: $\sigma_{A A}=1, \sigma_{B B}=5 / 6$, $\sigma_{A B}=\left(\sigma_{A A}+\sigma_{B B}\right) / 2, m_{B}=m_{A} / 2=1$, and $\varepsilon=1$. Lengths, times and temperatures are given in units of $\sigma_{A A}, \sqrt{m_{B} \sigma_{A A}^{2} / \varepsilon}$, and $\varepsilon / k_{B}$, respectively; we use $q_{0}$ to denote the wave vector of the first peak in the structure factor. In our simulations the total number of particles was $N=8000$, with $N_{A}=N_{B}=4000$. The use of the WCA reference potential makes this system computationally more efficient to simulate than the original Lennard-Jones one of Ref. [28]. A detailed study of the dynamics in the supercooled regime of this WCA mixture will be presented elsewhere [29]. The molecular dynamics simulations of this section conserve energy, and we use a range of energies at each temperature. This allows estimates of both canonical and microcanonical susceptibilities. Details are given in the Appendix; the data of Figs. 2, 4 is for the canonical susceptibility.

Figure 2 shows the self-intermediate scattering function $F_{\mathrm{s}}(k, t)$ at various temperatures $T=0.5$ to 0.36 , for the wave vector of the first peak of the structure factor $k=q_{0}$, and for a smaller wavevector $k=q_{0} / 4$. At the lowest temperature shown, $T=0.36$, the system is clearly in the supercooled regime, dynamics is heterogeneous, and the self-diffusion constant exceeds the value 
expected by the Stokes-Einstein relation by over an order of magnitude [29]. The four-point susceptibilities have the expected behaviour, becoming larger and peaking later the lower the temperature, as shown in the lower panels of figure 2 ,

Figure 3 concentrates on the lowest temperature we simulated, $T=0.36$. It shows the two point function $F_{\mathrm{S}}(k, t)$ for various wave vectors $k$. The growth of the distinct part of $\chi_{4}$ towards its peak can be fitted by a power of $t$. The fitted exponent is smaller than 1 for wave vectors near $q_{0}$, but closer to 1.5 for small $k$. The former result coincides with what was observed in similar systems [8], but the latter was not anticipated before. Interestingly, a similar $k$ dependence has been observed experimentally in a sheared granular material [11].

In section II we assumed that $\chi_{M}(a, t)$ is dominated by persistence correlations for small $a$, where displacement correlations are unimportant. At small times, the effect of increasing $a$ is to reduce the susceptibility. This is analogous to the effect of decreasing $k$ in $\chi_{4}(k, t)$. On the other hand, the $k$ dependence of the peak in $\chi_{4}(k, t)$ arises from the displacement correlations and will be absent (or at least much weaker) in $\chi_{M}(a, t)$. Both these predictions are consistent with the data of Fig. 4, calculated in the atomistic system of section III However, we note that the relative range of $a$ over which we have measured $\chi_{M}(a, t)$ is smaller than the range of $k$ used for $\chi_{4}(k, t)$. It may be that particle displacement correlations will be important at larger $a$. This effect can be estimated within the framework of section II It appears as an extra term in (15).

\section{KINETICALLY CONSTRAINED MODELS}

\section{A. Kinetically constrained lattice gas}

The kinetically constrained lattice gases of Jäckle and Krönig [18] provide simple caricatures of supercooled liquids. We consider the (2)-TLG in which hard core particles move on a triangular lattice: movement between sites and $i$ and $j$ is allowed only if both the mutual neighbours of sites $i$ and $j$ are empty. Relaxation in the model involves strongly co-operative motion and the relaxation time increases very rapidly with increasing density.

We show correlation functions in Fig. 5. We plot the self-intermediate scattering functions for particles at a high density $\rho=0.8$; we use wave vectors from $k=\pi$ to $k=\pi / 64$ (for details see Ref. [30]). We note that the exponents governing the growth of $\chi_{4}(k, t)$ in the (2)-TLG are rather similar to those in the atomistic system. These four-point functions are measured at constant density.

\section{B. Fredrickson-Andersen (FA) model}

The one-spin facilitated Fredrickson-Andersen (FA) model represents the extreme of coarse-graining in which
(2)-TLG, $\rho=0.8$
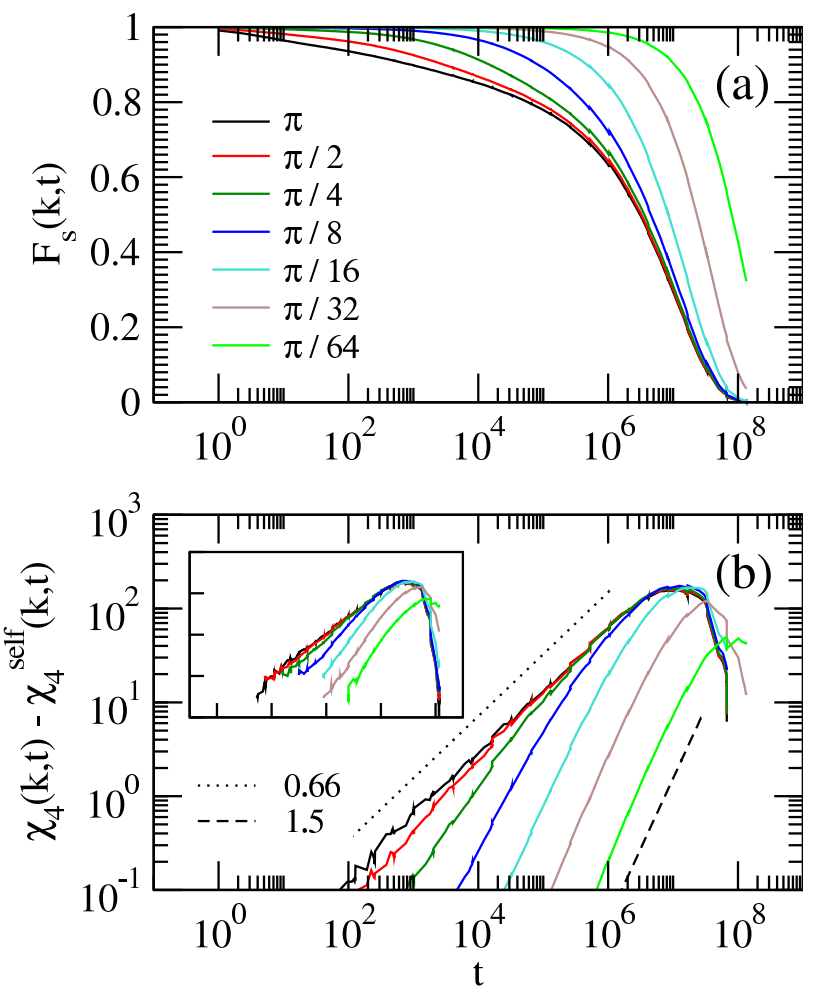

FIG. 5: (Color online) (a) Self-intermediate scattering function $F_{s}(k, t)$ of particles in the (2)-TLG model, at density $\rho=0.8$, for wave vectors $k=\pi, \pi / 2, \pi / 8, \pi / 16, \pi / 32, \pi / 64$. (b) Susceptibilities $\chi_{4}(k, t)$ for the self-correlators of the top panel. For large $k, \chi_{4}$ grows with an exponent close to 0.7, for small $k$ with an exponent close to 1.5. The inset shows the normalized function $\chi_{4} / \chi_{4}^{\text {self }}-1$ in the same scale as the main panel.

one conjectures that the only variables relevant for heterogeneity are binary labels for regions in which mobility is present. It is the simplest kinetically constrained model displaying both dynamic heterogeneity [4] and decoupling [13]. The system is a one dimensional chain in which mobile regions are represented by up spins and immobile regions by down spins. The energy of the system is simply the number of up spins: spins flip with Metropolis rates if and only if at least one neighbouring spin is up.

In order to consider particle motion in such a system, we couple probe particles to the spins as in [13]. Probes can hop only between adjacent mobile sites and such moves are attempted with rate unity. Probes do not interact with one another (more than one probe may occupy a single site). The stationary distribution of probes is uniform and uncorrelated with the spin variables.

Figure 6 (a) shows the self-intermediate scattering functions of probe molecules in the FA model at a low temperature $T=0.2$ for various wave vectors (the ensemble is again that in which the probe density is constant). For large wave vector the self-correlator is stretched and 

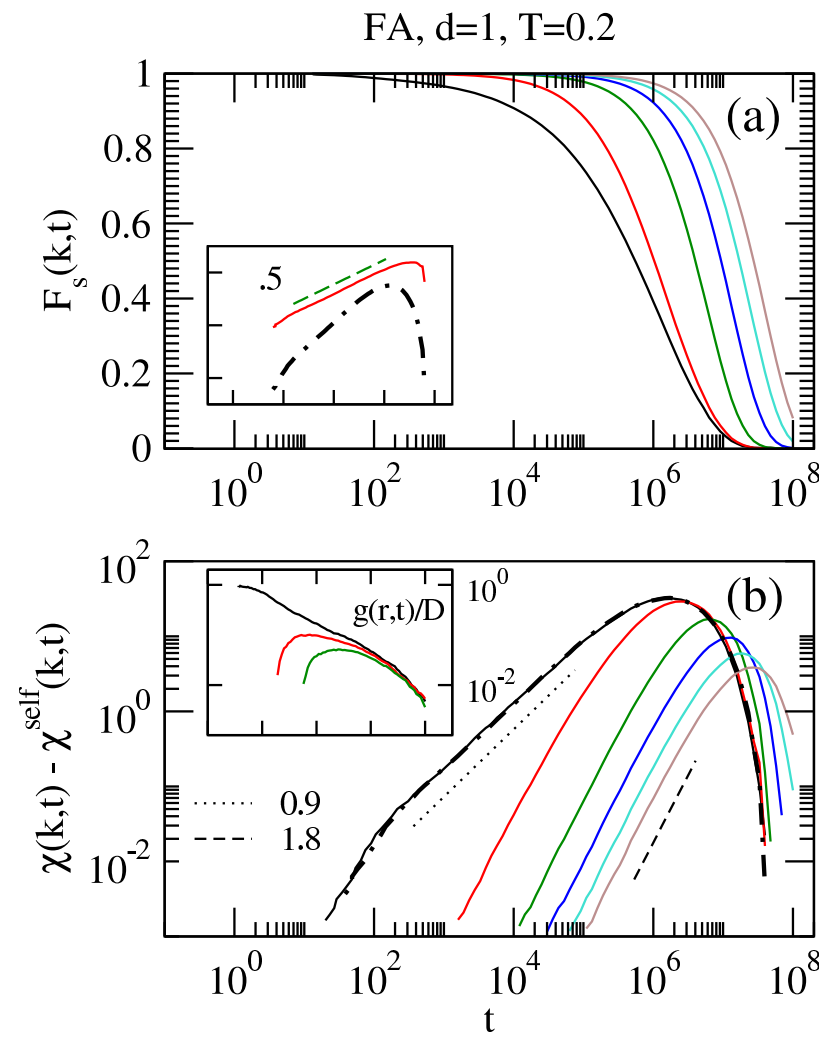

FIG. 6: (Color online) (a) Self-intermediate scattering function $F_{s}(k, t)$ of probes embedded in an FA model in $d=1$, at temperature $T=0.5$, for wave vectors $k=$ $\pi, \pi / 11, \pi / 31, \pi / 51, \pi / 71, \pi / 91$. The inset shows the susceptibility of the persistence given in Eq. (7) (dot-dashed) and the quantity $N_{\mathrm{p}}(t)$ (full), which grows approximately as $t^{1 / 2}$ (dashed). (b) Susceptibilities $\chi_{4}(k, t)$ for the self-correlators of the top panel. We show the non-trivial part, with the selfterm $\chi_{4}^{\text {self }}(k, t)$ removed. $\chi_{4}$ for $k=\pi$ follows the contribution of Eq. (7) (dot-dashed). For large $k, \chi_{4}$ grows with an exponent close to 1 , for small $k$ with an exponent close to 1.5. The inset shows the function $g(r, t)$, see Eq. (8), for inter particle distances $r=0,1,2$, in the same temporal scale as the main panel.

tracks the persistence function, while for small enough wave vectors self-correlators become exponential, according to Eq. (4) (see Ref. [15] for details). The inset shows the time dependence of $N_{\mathrm{p}}(t)$, as extracted from the susceptibility of the persistence, see Eq. (7). Figure 6 (b) shows the corresponding susceptibilities $\chi_{4}(k, t)$. We note that the exponent governing the increase of $\chi_{4}(k, t)$ is near to unity for large $k$, as reported in [8]; however the exponent at small $k$ is closer to 1.5 . In the notation of (11) we have $N_{\mathrm{p}}(t) \sim[1-P(t)] \sim t^{1 / 2}$ at large $k$ which accounts for the exponent of unity in that regime. The inset to the bottom panel shows the function $g(r, t)$, for $r=0,1,2$. As $t$ increases the distance $r$ over which these correlations are significant also increases. This is consistent with our assumption of an increasing function $N_{g}(t)$ in section $\llbracket$.

\section{Acknowledgments}

We have benefited from discussions on this topic with David Reichman, Ludovic Berthier, Hans Andersen, Phill Geissler, Dan Goldman, and Eric Weeks. This work was supported by EPSRC grants no. GR/R83712/01 and GR/S54074/01 (JPG); University of Nottingham grant no. FEF 3024 (JPG); NSF grant CHE-0543158 (RLJ); and by the US Department of Energy Grant no. DEFG03-87ER13793 (DC).

\section{APPENDIX: ENSEMBLE DEPENDENCE OF $\chi_{4}$}

Recently, Berthier et al. [26] suggested that the difference between $\chi_{4}(k, t)$ in two different ensembles provides a good estimate of the value of $\chi_{4}(k, t)$ itself. In this Appendix we discuss the ensemble dependence of the four point susceptibility in the models that we consider. Not surprisingly, we find that the difference in $\chi_{4}(k, t)$ between ensembles has a significant wavelength dependence. At the lowest temperature we simulated for the WCA mixture, and the highest density for the (2)-TLG, we find that the method of [26] does not give accurate numerical estimates of the susceptibility, although it does give the correct order of magnitude for $\chi_{4}(k, t)$ at times for which that function is near its maximum, the estimate working better for smaller $k$ than for larger $k$. It would be interesting to see whether the estimate of [26] becomes better or worse with decreasing temperature, but this is beyond the scope of this appendix.

In terms of equation (1), the ensemble dependence of $\chi_{4}(k, t)$ is contained in the definition of the average required by the angle brackets. This average is over any stochasticity in the dynamics, and over an initial condition that is specified by the choice of ensemble. The key point is that $\chi_{4}(k, t)$ measures fluctuations in an observable that couples with equal weight to every particle in the system. So the presence of a global constraint (on the total energy of the system, for example) suppresses these fluctuations. By contrast, the quantity $G_{4}(\boldsymbol{q}, \boldsymbol{k}, t)$ measures fluctuations on finite length scales, as long as $\boldsymbol{k}$ and $\boldsymbol{q}$ are both finite. In that case, taking the thermodynamic limit leads to a value of $G_{4}(\boldsymbol{q}, \boldsymbol{k}, t)$ that is independent of global constraints.

The effect of global constraints on $\chi_{4}(k, t)$ can be measured directly in the (2)-TLG, by considering the susceptibility in both canonical and grand canonical ensembles. We denote the canonical susceptibility by $\chi_{4}^{(N)}(k, t)$, which is defined as in (11), with the averages over the dynamics, and over all initial conditions with exactly $N$ particles. For grand canonical systems, we promote $N$ to a fluctuating quantity (operator), and write:

$$
\chi_{4}^{(\mu)}(k, t)=\langle\hat{N}\rangle^{-1}\left\langle\left|\sum_{j=1}^{\hat{N}}\left(\hat{F}_{j}(\boldsymbol{k}, t)-F_{\mathrm{S}}(k, t)\right)\right|^{2}\right\rangle \text {. }
$$

where the average is over the dynamics, and over initial 

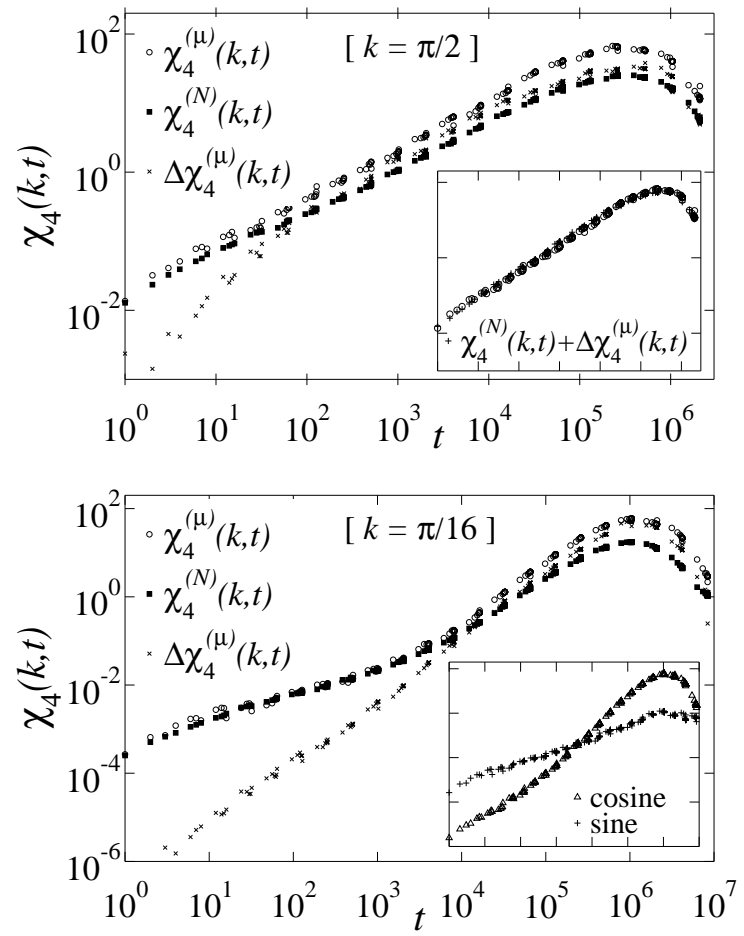

FIG. 7: (a) Figure showing $\chi_{4}(k, t)$ in the (2)-TLG at $\rho=0.77$ and $k=\pi / 2$ in both canonical and grand canonical ensembles, together with the difference term $\Delta \chi_{4}^{(\mu)}(k, t) \equiv$ $\rho^{2}(1-\rho)\left[\partial F_{\mathrm{S}}(k, t) / \partial \rho\right]^{2}$. The contribution of $\Delta \chi_{4}^{(\mu)}(k, t)$ to $\chi_{4}^{(\mu)}(k, t)$ is comparable to that of $\chi_{4}^{(N)}(k, t)$. (Inset, a) Illustration that the grand canonical susceptibility satisfies the relation A.2. The scales are those of the main panel. (b) Similar data in the (2)-TLG at $k=\pi / 16$. For smaller wave vectors, such as these, the difference term dominates the peak of the grand canonical susceptibility. (Inset, b) Two contributions to the grand canonical susceptibility: see A.4. At early times, the susceptibility is dominated by the ensemble independent sine part, while the peak is dominated by the cosine part. The scales are again those of the main panel.

conditions with all particle numbers, with weights set by the chemical potential $\mu$.

These susceptibilities are related to each other by [27]

$$
\begin{aligned}
\chi_{4}^{(\mu)}(k, t) & =\chi_{4}^{(N)}(k, t)+\Delta \chi_{4}^{(\mu)}(k, t), \\
\Delta \chi_{4}^{(\mu)}(k, t) & \equiv \rho V\left\langle(\delta \rho)^{2}\right\rangle\left[\frac{\partial F_{\mathrm{s}}(k, t)}{\partial \rho}\right]^{2},
\end{aligned}
$$

where $\rho$ is the density and $V$ the volume of the system; the average is grand canonical. The density fluctuations of the (2)-TLG are those of a lattice gas, so that $V\left\langle(\delta \rho)^{2}\right\rangle=\rho(1-\rho)$.

The relative sizes of the two terms on the right hand side of (A.2) depend on the strength of the coupling of the dynamics to density fluctuations in the system. Berthier et al. argued [26] that the second term tends to dominate the grand canonical susceptibility in athermal glassy systems. Since the thermodynamic properties of the (2)-
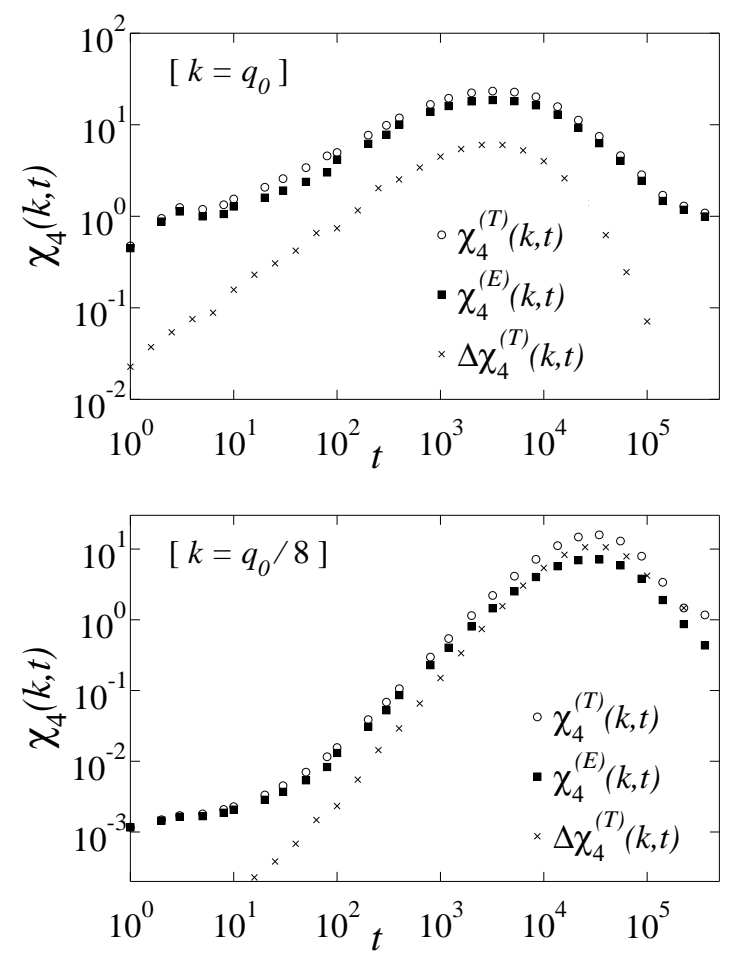

FIG. 8: (a) Figure showing four point susceptibilities in the WCA mixture at $T=0.36$ and $k=q_{0}$, and the difference term $\Delta \chi_{4}^{(T)}(k, t)$. The difference term is significantly smaller than the canonical susceptibility. (b) Similar data at $k=q_{0} / 8$. In this case the difference term exceeds the microcanonical susceptibility. The data is consistent with the exact relation A.10.

TLG system are trivial, $\chi_{4}(k, t)$ can be measured in both ensembles, and the prediction for the difference can also be evaluated. Results are shown in figure [7, at a high density $\rho=0.77$. The difference between ensembles is consistent with the prediction of (A.2). We find that the two contributions to $\chi_{4}^{(\mu)}(k, t)$ are of similar size for large wave vectors, while the second term dominates at small wave vectors. In both cases, the form of the second term is similar to that of $\chi_{4}^{(\mu)}(k, t)$ for times near the peak of the susceptibility.

Our definition of $\chi_{4}(k, t)$ includes both the sine and cosine parts of the operator $\hat{F}_{j}(k, t)=\cos \left[\boldsymbol{k} \cdot \Delta \hat{\boldsymbol{r}}_{j}(t)\right]+$ $i \sin \left[\boldsymbol{k} \cdot \Delta \hat{\boldsymbol{r}}_{j}(t)\right]$, leading to two contributions to $\chi_{4}(k, t)$. That is,

$$
\chi_{4}(k, t)=\chi_{4}^{\cos }(k, t)+\chi_{4}^{\sin }(k, t)
$$

with

$$
\begin{aligned}
& \chi_{4}^{\cos }(k, t)=\langle\hat{N}\rangle^{-1}\left\langle\left\{\sum_{j} \cos \left[\boldsymbol{k} \cdot \Delta \hat{\boldsymbol{r}}_{j}(t)\right]-F_{\mathrm{s}}(k, t)\right\}^{2}\right\rangle \\
& \chi_{4}^{\sin }(k, t)=\langle\hat{N}\rangle^{-1}\left\langle\left\{\sum_{j} \sin \left[\boldsymbol{k} \cdot \Delta \hat{\boldsymbol{r}}_{j}(t)\right]\right\}^{2}\right\rangle,
\end{aligned}
$$


The fluctuations in the sine part are ensemble independent, since the average of this part is zero. Hence, the difference term $\Delta \chi_{4}^{(\mu)}(k, t)$ is bounded above by the cosine part of $\chi_{4}^{(\mu)}(k, t)$; it follows that the difference term is qualitatively different from the total susceptibility when the total susceptibility is dominated by the sine part. This is the case at small wave vectors and small times, as shown in the inset of the lower panel of figure 7 .

We can make a similar analysis of the WCA mixture. For each temperature, the data of section [II] was obtained using microcanonical simulations at eight different values of the energy, sampled from the appropriate canonical ensemble. This allows estimates of both microcanonical and canonical susceptibilities, which we denote by $\chi_{4}^{(E)}(k, t)$ and $\chi_{4}^{(T)}(k, t)$ respectively. The microcanonical susceptibility for a given energy is given by (1), with microcanonical averages throughout. In particular, we must use

$$
\delta \hat{F}_{j}(k, t)=\hat{F}_{j}(k, t)-\left\langle\hat{F}_{j}(k, t)\right\rangle_{\text {microcanonical }},
$$

where the average involves data at a single energy. Having used this prescription to calculate $\chi_{4}^{(E)}(k, t)$ for each energy, we then average $\chi_{4}^{(E)}(k, t)$ over the eight representative energies at each temperature. Since the fluctuations in $\chi_{4}^{(E)}(k, t)$ itself are small, the resulting average is an unbiased estimate of the microcanonical susceptibility at the (canonical) average energy associated with that temperature. For the canonical susceptibility, we use (1), with canonical averages throughout, including

$$
\delta \hat{F}_{j}(k, t)=\hat{F}_{j}(k, t)-\left\langle\hat{F}_{j}(k, t)\right\rangle_{\text {canonical }} .
$$

We estimate canonical averages by averaging over the data from the eight energies that are representative of the relevant temperature.

The canonical and microcanonical susceptibilities satisfy [27]

$$
\begin{aligned}
\chi_{4}^{(T)}(k, t) & =\chi_{4}^{(E)}(k, t)+\Delta \chi_{4}^{(T)}(k, t) \\
\Delta \chi_{4}^{(T)}(k, t) & =\left(k_{B} T^{2} / c_{V}\right)\left(\partial F_{\mathrm{s}}(k, t) / \partial T\right)^{2}(\mathrm{~A} .10)
\end{aligned}
$$

where $c_{V}$ is the specific heat per particle at constant volume. The behavior of $c_{V}$ that we have computed for the WCA mixture is unremarkable. It rises slowly with lowering temperature, indicative of mean-square potential energy and kinetic energy fluctuations that are virtually independent of temperature over the lowest temperatures we have considered.

We show results for both susceptibilities in figure 8 , together with an estimate of $\Delta \chi_{4}^{(T)}(k, t)$. [In evaluating $\Delta \chi_{4}^{(T)}(k, t)$, the derivative and specific heat are evaluated by a finite difference analysis of the temperature dependence of the the mean energy and $\left.F_{\mathrm{S}}(k, t)\right]$. The data is at the low temperature $T=0.36$. At large $k, \chi_{4}^{(T)}(k, t)$ is not saturated by the difference term $\Delta \chi_{4}^{(T)}(k, t)$. Instead the microcanonical contribution is larger. However, at smaller $k$, the difference term exceeds the microcanonical part for times near the peak. Just as in the (2)-TLG, the difference term becomes more significant at smaller $k$, and at later times. Since the size of the difference term reflects coupling between energy fluctuations and dynamics, it would appear that motion over large distances couples strongly to the local value of the energy while local rearrangements do not.

Very recently, Szamel and Flenner [31] measured the contribution of global energy fluctuations to the canonical susceptibility $\tilde{\Delta} \chi_{4}^{(T)}(k, t)=$ $\left(k_{B} T^{2} / c_{V}^{\text {pot }}\right)\left(\partial F_{\mathrm{s}}(k, t) / \partial T\right)^{2}$, in a model glass-former with Brownian dynamics (here, $c_{V}^{\text {pot }}$ is the potential energy contribution to the heat capacity, at constant volume). Since Brownian dynamics are non-conservative, $\tilde{\Delta} \chi_{4}^{(T)}(k, t)$ does not represent a bound on the canonical $\chi_{4}(k, t)$. However, the results of [31] show that while $\tilde{\Delta} \chi_{4}^{(T)}(k, t)$ and the unconstrained susceptibility are not equal, they are of the same order of magnitude for large $k$. We find a similar situation in the (2)-TLG; in the WCA mixture then the difference term $\Delta \chi_{4}^{(T)}(k, t)$ is much smaller than the canonical susceptibility at these wave vectors.
[1] For reviews see: H. Sillescu, J. Non-Cryst. Solids 243, 81 (1999); M.D. Ediger, Annu. Rev. Phys. Chem. 51, 99 (2000); S.C. Glotzer, J. Non-Cryst. Solids, 274, 342 (2000); R. Richert, J. Phys. Condens. Matter 14, R703 (2002); H. C. Andersen, Proc. Natl. Acad. Sci. U. S. A. 102, 6686 (2005).

[2] R. Yamamoto and A. Onuki, Phys. Rev. E 58, 3515 (1998).

[3] C. Bennemann, C. Donati, J. Baschnagel, and S. C. Glotzer, Nature 399, 246 (1999).

[4] J. P. Garrahan and D. Chandler, Phys. Rev. Lett. 89, 035704 (2002).

[5] G. Biroli and J. P. Bouchaud, Europhys. Lett. 67, 21
(2004); G. Biroli and J. P. Bouchaud, K. Miyazaki, D. R. Reichman, cond-mat/0605733

[6] S. Franz, C. Donati, G. Parisi, and S. C. Glotzer, Philos. Mag. B 79, 1827 (1999).

[7] L. Berthier, Phys. Rev. E 69, 020201 (2004).

[8] C. Toninelli, M. Wyart, L. Berthier, G. Biroli, and J. P. Bouchaud, Phys. Rev. E 71, 041505 (2005).

[9] In, e.g., Ref. [8], only the fluctuations of the real part of $\delta F(k, t)$ were considered. We prefer to consider the full phase factor since both real and imaginary parts contribute to the Fourier transform of the density; both parts contribute to our $\chi_{4}(k, t)$. Numerical contributions of the two components of of $\chi_{4}(k, t)$ are similar, except when ei- 
ther of $k$ or $t$ is very large or very small.

[10] N. Lacevic, F.W. Starr, T.B. Schrøder and S.C. Glotzer, J. Chem. Phys. 119, 7372 (2003).

[11] O. Dauchot, G. Marty, and G. Biroli, Phys. Rev. Lett. 95, 265701 (2005).

[12] F. Ritort and P. Sollich, Adv. Phys. 52, 219 (2003).

[13] Y.-J. Jung, J. P. Garrahan, and D. Chandler, Phys. Rev. E 69, 061205 (2004).

[14] Y.-J. Jung, J. P. Garrahan, and D. Chandler, J. Chem. Phys. 123, 084509 (2005).

[15] L. Berthier, D. Chandler, and J. P. Garrahan, Europhys. Lett. 69, 320 (2005).

[16] L. Maibaum, Ph.D. thesis, University of California Berkeley (2005).

[17] J. D. Weeks, D. Chandler, and H. C. Andersen, J. Chem. Phys. 54, 5237 (1971).

[18] J. Jäckle and A. Krönig, J. Phys.-Condens. Matter 6, 7633 (1994).

[19] G. H. Fredrickson and H. C. Andersen, Phys. Rev. Lett. 53, 1244 (1984).

[20] See e.g.: F. Fujara, B. Geil, H. Sillescu and G. Fleischer, Z. Phys. B-Condens. Mat. 88, 195 (1992); M.T. Cicerone and M.D. Ediger, J. Chem. Phys. 104, 7210 (1996); S.F.
Swallen, P.A. Bonvallet, R.J. McMahon and M.D. Ediger, Phys. Rev. Lett. 90, 015901 (2003).

[21] E. W. Montroll and G. H. Weiss, J. Math. Phys. 6, 167 (1965).

[22] W. Gotze, J. Phys. Condens. Matter 11, A1 (1999).

[23] M. Fuchs, W. Gotze, and M. R. Mayr, Phys. Rev. E 58, 3384 (1998).

[24] G. Foffi, W. Gotze, F. Sciortino, P. Tartaglia, and T. Voigtmann, Phys. Rev. E 69, 011505 (2004).

[25] E. Flenner and G. Szamel, Phys. Rev. E 72, 031508 (2005).

[26] L. Berthier, G. Biroli, J.-P. Bouchaud, L. Cipelletti, D. E. Masri, D. L'Hote, F. Ladieu, and M. Pierno, Science 310, 1797 (2005).

[27] J. L. Lebowitz, J. K. Percus, L. Verlet, Phys. Rev. 153, 250 (1967).

[28] G. Wahnstrom, Phys. Rev. A 44, 3752 (1991).

[29] L. Maibaum, D. Chandler and J.P. Garrahan, in preparation (2006).

[30] A. C. Pan, J. P. Garrahan, and D. Chandler, Phys. Rev. E 72, 041106 (2005).

[31] G. Szamel and E. Flenner, cond-mat/0605244. 